\title{
On the determination of neutron multiplication by the Rossi-alpha method*
}

\author{
Vladimir A. Grabezhnoy ${ }^{1}$, Viktor A. Dulin ${ }^{1}$, Vitaliy V. Dulin ${ }^{1}$, Gennady M. Mikhailov ${ }^{1}$ \\ 1 JSC SSC RF-IPPE n.a. A.I. Leypunsky, 1 Bondarenko Sq., 249033 Obninsk, Kaluga Reg., Russia \\ Corresponding author: Vitaliy V. Dulin (dulin@ippe.ru)
}

Academic editor: Georgy Tikhomirov • Received 09 December 2020 • Accepted 1 July 2021 • Published 23 September 2021

Citation: Grabezhnoy VA, Dulin VA, Dulin VV, Mikhailov GM (2021) On the determination of neutron multiplication by the Rossialpha method. Nuclear Energy and Technology 7(3): 253-257. https://doi.org/10.3897/nucet.7.74156

\begin{abstract}
Introduction. This work contains the results of determining the prompt neutron multiplication factor in the subcritical state of a one-core BFS facility, obtained by the neutron coincidence method, for which the influence of the error in the $\beta_{\text {eff }}$ in determining the multiplication factor turned out to be insignificant. The core of the facility consisted of rods filled with pellets of metallic depleted uranium, $37 \%$ enriched uranium dioxide and $95 \%$ enriched plutonium, sodium, stainless steel and $\mathrm{Al}_{2} \mathrm{O}_{3}$. Stainless steel served as a reflector.

Methods. In contrast to the inverse kinetics equation solving (IKES) method, which is convenient for determining reactor subcritical states, the neutron coincidence method practically does not depend on the error in the value of the effective fraction of delayed neutrons $\beta_{\text {eff }}$. If in the IKES method the reactivity value is obtained in fractions of $\beta_{\text {effr }}$, i.e., from the measurement of delayed neutrons, the neutron coincidence method is based on the direct measurement of the value $\left(1-k_{\sigma p}\right)^{2}$, where is the effective multiplication factor by prompt neutrons. The total multiplication factor is defined as $k_{\mathrm{eff}}=k_{\sigma p}+\beta_{\mathrm{eff}}$. If, for example, $k_{\mathrm{eff}} \approx 0.9$ (which is typical for determining the fuel burnup campaign), then it is the error in determining $k_{\sigma p}$ that is the main one in comparison with the error in $\beta_{\text {eff }}$. Thus, a $10 \%$ error in $\beta_{\text {eff }}$ of 0.003-0.004 (typical for plutonium breeders) will make a contribution to the error $1-k_{\text {eff }}$ equal to $1-k_{\sigma p}+\beta_{\text {eff }} \approx$ 0.00035 , i.e., approximately $0.35 \%$, but not $10 \%$, as in the IKES method.

Rossi-alpha measurements were carried out using two ${ }^{3} \mathrm{He}$ counters and a time analyzer. The measurement channel width $\Delta t$ was $1.0 \mu \mathrm{s}$. From these measurements, the value of the prompt neutron multiplication factor was obtained. In this case, the space-isotope correlation factor for the medium with a source was calculated using the following values: $\Phi(x)$ - solutions of the inhomogeneous equation for the neutron flux and $\Phi^{+}(x)$ - solutions of the ajoint inhomogeneous equation.

Results. The authors also present a comparison of the results of the Rossi-alpha experiment and measurements of the BFS-73 subcritical facility by the standard IKES method in determining the multiplication factor value. The data of the IKES method differ insignificantly from the results of the Rossi-alpha method over the entire range of changes in the subcriticality with an increase in the subcriticality of the BFS-73 one-core facility.

Conclusion. It was impossible to apply the neutron coincidence method to fast reactors; however, the method turned out to be quite workable on their models created at the BFS facility, which was successfully demonstrated in this study.
\end{abstract}

\section{Keywords}

Neutron coincidence method, Rossi- $\alpha$ method, spatial-isotope correlation factor, prompt neutron multiplication factor

* Russian text published: Izvestiya vuzov. Yadernaya Energetika (ISSN 0204-3327), 2021, n. 2, pp. 50-58. 


\section{Introduction}

The multiplication factor, estimated by the standard methods adopted at the BFS facility, i.e., the IKES method (Kazansky and Matusevich 1994) at shallow subcriticality $\left(\sim \beta_{\text {eff }}\right)$, and the inverse multiplication method, taking into account the change in the neutron registration efficiency at deeper subcriticality $\left(\sim 10 \beta_{\text {eff }}\right)$, is directly related to the accuracy of measurements of the $\beta_{\text {eff }}$ value itself. Previously implemented international programs for measuring $\beta_{\text {eff }}$ (see, for example, (Dulin VA and Dulin VV 1998)) in fairly simple single-zone cores with dense reflectors made of uranium dioxide made it possible to determine the error in $\beta_{\text {eff }} \approx 3-4 \%$. Nevertheless, it is of interest to determine deep subcriticalities by a fundamentally different method, without using $\beta_{\text {eff }}$

The work contains the results of determining the multiplication factor in the subcritical state, obtained by the neutron coincidence method (Dulin and Matvienko 2002), for which the influence of the error in $\beta_{\text {eff }}$ in determining the multiplication factor is insignificant (tens of times less than in methods where the value of reactivity is inversely proportional to the value of $\beta_{\text {eff, }}$ as in the IKES method (Kazansky and Matusevich 1994)).

\section{BFS facility measurements}

The effective neutron multiplication factor in the subcritical state of a single-core BFS facility was determined using both of the above methods. The core of the facility consisted of rods filled with pellets of metallic depleted uranium, 37\% enriched uranium dioxide and 95\% enriched plutonium, sodium, stainless steel and $\mathrm{Al}_{2} \mathrm{O}_{3}$. Stainless steel served as a reflector. The height of the core was $45 \mathrm{~cm}$, the equivalent diameter $\approx 65 \mathrm{~cm}$. The subcritical state was achieved by removing from the core 11 fuel rods, of which the controls and CPS were composed.

Measurements by the neutron coincidence method were carried out using two SNM-18 ${ }^{3} \mathrm{He}$ counters placed in the central channel (instead of a fuel rod) and a time analyzer (TA). The helium pressure in the counters was about two atmospheres. One of the counters was located $1 \mathrm{~cm}$ above the central plane of the critical facility core, and the other was below it. The length of the counter was approximately $25 \mathrm{~cm}$. The first was used as a starting one for the TA, and the second was used as a counting one. The width of the VA channel $\Delta t=1 \mu \mathrm{s}$. The delay $T$ in the counting channel was measured and turned out to be $40.1 \mu \mathrm{s}$.

As an example, Figure 1 presents the result of one of such measurements and Figure 2 shows the temporal distribution of its correlated part obtained after the constant background was subtracted (determined in the region of 125-250 channels). Along the abscissa, the TA channels (1 $\mu$ s wide) are located; the ordinate shows the count in them.

In (Mikhailov and Dulin 1995), the analysis method is described in detail, which makes it possible, using the

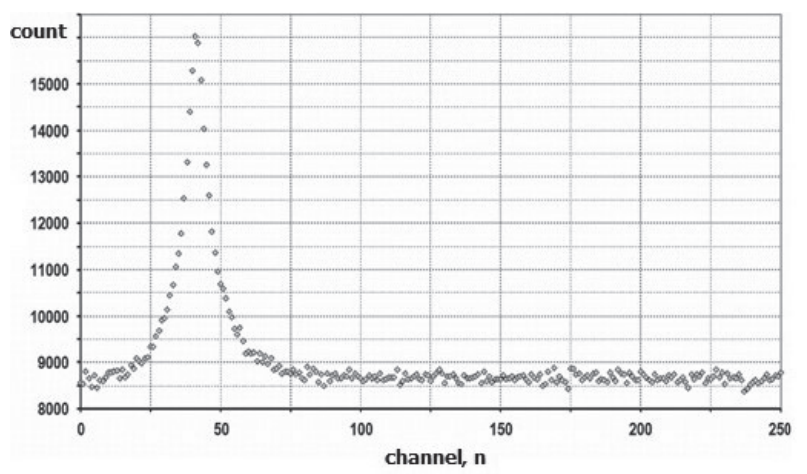

Figure 1. Time distribution of the neutron counting rate.

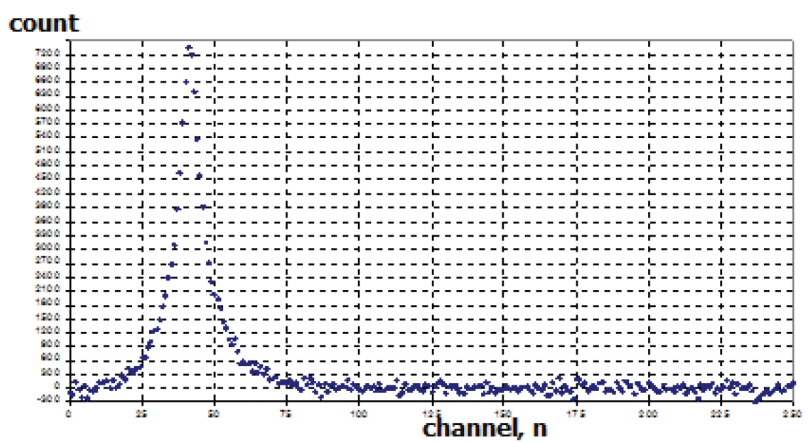

Figure 2. Correlated part of the temporal distribution of the neutron counting rate.

measured correlated part of the distributions (see Fig. 2), the average constant background $\bar{N}$ (see Fig. 1, to the right of the $125^{\text {th }}$ channel), the integral of readings above zero in the right of the correlated part of the distribution (see Fig. 2) $J_{\text {right }}$ and the source of spontaneous fissions $Q_{\mathrm{sp}}$, to obtain the value of the prompt neutron multiplication factor $\left(1-k_{\sigma p \exp }\right)^{2}$ :

$$
\left[N /\left(2 J_{\text {right }} \Delta t Q_{\mathrm{sp}}\right)\right]_{\exp } D=\left(1-k_{\sigma p \exp }\right)^{2},
$$

where $D$ is the calculated spatial-isotope correlation factor for the medium with the source:

$$
D=\left\langle Q_{c f}(x)\right\rangle \frac{\langle\Theta\rangle}{\langle\Xi\rangle^{2}}
$$

$$
\begin{gathered}
\Theta=\left[\left(4.91 \sum_{f 235}(x)+7.06 \sum_{f 239}(x)+5.28 \sum_{f 238}(x)\right) \Phi(x)+\right. \\
\left.3.825 Q_{s p}(x)\right]\left(\Phi^{+}(x)\right)^{2} ; \\
\Xi=\left[\left(2.50 \sum_{f 235}(x)+2.98 \sum_{f 239}(x)+2.59 \sum_{f 238}(x)\right) \Phi(x)+\right. \\
\left.2.156 Q_{s p}(x)\right] \Phi^{+}(x)^{2},
\end{gathered}
$$

where $\Phi(x)$ is the solution to the inhomogeneous equation for the neutron flux; $\Phi^{+}(x)$ is the fission spectrum-averaged solution to the ajoint inhomog eneous equation, which is the probability for the detector to register a neutron that appears at the point $x$ (Marchuk and Orlov 1961). The numbers in the denominator $\Xi$ are the $v$ values for fissions of ${ }^{235} \mathrm{U},{ }^{239} \mathrm{Pu},{ }^{238} \mathrm{U}$ and the source of spontaneous fissions of ${ }^{240} \mathrm{Pu}$, and those in the numerator $\Theta$ are the $\langle v(v-1)\rangle$ values for themselves, respectively. 
The processing of Rossi- $\alpha$ temporal distributions was carried out taking into account the asymmetry of the temporal distribution of the background relative to the center of the correlated signal, which was at $40.1 \mu \mathrm{s}$ of the TA scale. For this, the signal of a periodic pulse generator with a duration of $0.05 \mu$ s was applied simultaneously to the starting and counting (through the delay line T) TA channels with a channel width of $0.1 \mu \mathrm{s}$, and the count was recorded in the $401^{\text {st }}$ channel, which corresponded to the delay value $T=40.1 \mu \mathrm{s}$.

This type of background asymmetry was found experimentally on a fast critical assembly (Tsutomu 1968), and a quantitative description of the time dependence of the background $N(t)$ was given in (Mikhailov and Dulin 1995). Having carried out measurements with exact knowledge of the delay $T$, but not assuming, as was done in (Grabezhnoy et al. 2015), that it is determined by the position of the peak maximum visible on the screen, we compared the results of both approaches and experimentally verified the theoretical conclusions (Mikhailov and Dulin 1995), according to which:

$$
\begin{gathered}
N_{\text {left }}(t)=N\left\{1-A \frac{C \alpha}{\alpha+C}\left[\frac{2 \alpha}{\alpha-C} \cdot \mathrm{e}^{C(t-T)}-\frac{\alpha+C}{\alpha-C} \cdot \mathrm{e}^{\alpha(t-T)}\right]\right\} ; t<T,(2) \\
N_{\text {right }}(t)=N\left\{1-A \frac{C \alpha}{\alpha+C} \cdot \mathrm{e}^{-\alpha(t-T)}\right\} ; t \geq T
\end{gathered}
$$

Note that the temporal distribution of the background is continuous, i.e., $N_{\text {left }}(T)=N_{\text {right }}(T)$.

The correlated part of the distribution at $t \geq T$ obtained after the constant mean background is subtracted, equal to $[A C \alpha /(\alpha+C)] \mathrm{e}^{-\alpha(t-T)}$ (the right side visible in the experiment), according to (Mikhailov and Dulin 1995), has an amplitude in $\alpha /(\alpha+C)$ times less than the theoretical value. Adding the 'lost' part of the background (3), we obtain the true distribution of the right side:

$$
R_{\text {cor }}(t)=A C_{\text {count }} C \mathrm{e}^{-\alpha(t-T)} ; t \geq T .
$$

Correspondingly, the area under the right correlated component (integral over $t \geq T$ ) is

$$
J_{\text {right }}=A C_{\text {count }} C / \alpha
$$

To obtain the true measured correlated areas $J_{\text {cor }}$ from those 'visible' above the constant background level $N$, it is necessary to additionally measure the speed of incoming counts $C$ at the start and then find the $\alpha$ value from the experiment. According to (7), it is on the right side of the equality $R_{\text {cor }}{ }^{\exp }(t)$ (see Figs 2,3), since the theoretical temporal behavior of the correlated component and the background 'under it' on the right have the same exponential dependence. Figure 3 shows that the decay time of the neutron density $\tau$ is approximately $8 \mu$ s.

Knowing $C$ and $\alpha$, we can calculate the value of the addition to the area $A C / \alpha$ to the right of $t \geq T$ :

$$
\Delta J_{\text {right }}=(A C / \alpha) \times[C /(\alpha+C)] .
$$

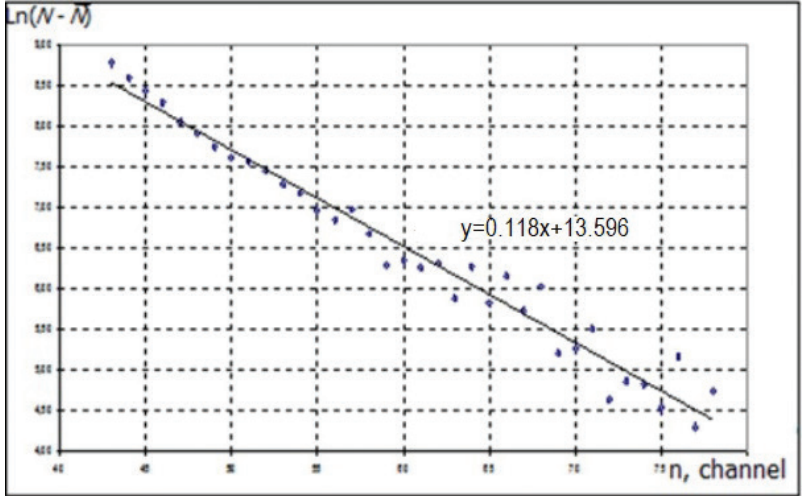

Figure 3. Time distribution of the correlated part obtained after the constant background is subtracted $\bar{N}$ (Axis Y).

When calculating $\Delta J_{\text {left }}(t)$, the integration, according to (Mikhailov and Dulin 1995), is taken for all $t<T$. Then the addition to the left side of the square will be

$$
\Delta J_{\text {left }}=-\frac{A C}{\alpha} \frac{C}{(\alpha+C)}\left[\frac{2 \alpha^{2}}{(\alpha-C) \tilde{N}}-\frac{\alpha+C}{(\alpha-C) \alpha}\right]
$$

Table 1 shows the results of the experiment (the first four columns), Table 2 uses the account of experimental additions in formulas (6), (7) and the values of the left side of formula (1), where $J_{\text {right }}$ and $J_{\text {left }}$ are taken from the fifth and sixth columns of Table 1. It can be seen that these experimental

\begin{tabular}{|c|c|c|c|c|c|}
\hline Files & $\begin{array}{c}\text { Starts C, } \\
\mathrm{s}-1\end{array}$ & $\begin{array}{c}\text { Channel background } \\
\text { on the right }\end{array}$ & $\alpha \times 10^{5}, s^{-1}$ & $J_{\text {left }}$ & $J_{\text {right }}$ \\
\hline RT-1 & $1544 \pm 44$ & 8666 & 1.191 & 36811 & 73436 \\
\hline RT-21 & $1816 \pm 46$ & 13836 & 1.002 & 56401 & 121326 \\
\hline RT-3 & $1796 \pm 15$ & 14993 & 1.119 & 62041 & 130442 \\
\hline
\end{tabular}
additions confirm the description of the time-background dependence $N(t)$ obtained in (Mikhailov and Dulin 1995) and are essential in terms of determining the total area $J$.

Table 1. Experimental results, taking into account the experimental additives

Table 2. Comparison of the results of the Rossi- $\alpha$ and IKES methods

\begin{tabular}{lcccc}
\hline Files & $\boldsymbol{J}_{\text {left }}+\boldsymbol{\Delta} \boldsymbol{J}_{\text {left }}$ & $\boldsymbol{J}_{\text {right }}+\boldsymbol{\Delta} \boldsymbol{J}_{\text {right }}$ & $\boldsymbol{N} /\left(\boldsymbol{J}_{\text {right }} \boldsymbol{\Delta t Q}\right)$ & $\boldsymbol{N} /\left[\left(\boldsymbol{J}_{\text {right }}+\boldsymbol{J}_{\text {leff }}\right) \boldsymbol{\Delta t} \boldsymbol{Q}\right]$ \\
\hline RT-1 & 73150 & 74376 & 0.0859 & 0.0858 \\
RT-21 & 111799 & 123485 & 0.0820 & 0.0869 \\
RT-3 & 123102 & 132503 & 0.0831 & 0.0859 \\
& & & $0.0837 \pm 0.0017$ & $0.0862 \pm 0.0005$ \\
& & $0.9624 \pm 0.0008-$ & $0.9630 \pm 0.0003-$ \\
$k_{\text {eff }}($ Rossi- $\alpha)-k_{\text {eff }}($ IKES $)$ & & $-0.9595 \pm 0.0030=$ & $-0.9595 \pm 0.0030=$ \\
& & & $=0.0029$ & $=0.0035$ \\
\hline
\end{tabular}

The source of spontaneous fissions $Q_{\text {sp }}$ was the ${ }^{240} \mathrm{Pu}$ source, which was included in the plutonium fuel. Using the calculated factor $D=0.0472$ and the effective source of spontaneous fissions ${ }^{240} \mathrm{Pu} Q_{\mathrm{sp}}=(7.64 \pm 0.21) \times 10^{6} \mathrm{n} / \mathrm{s}$ $(1 \sigma)$, we obtain the prompt neutron multiplication factor $1 /\left(1-k_{\sigma p \exp }\right)$ and the values $k_{\text {eff }}\left(\varphi_{\sigma}^{+}\right)=k_{\sigma p \exp }+\beta_{\text {eff }}$. In this method the absolute error $\beta_{\text {eff }}$ but not the relative error, as in the $\left|\rho_{s}\right|$-subcriticality (in units of $\beta_{\text {eff }}$ ) of the assembly. The difference between the $k_{\text {eff }}$ values obtained by different methods is shown in the last row of the Table 2. 
Table 3. The results of using the experiment of Rossi- $\alpha$ and measurements of the subcritical assembly by the IKES method

\begin{tabular}{|c|c|c|c|c|c|c|c|}
\hline File & $\alpha \times 10^{4}$ & $S_{\text {right }}$ in range channels (ch) & $S_{\text {left }}$, in range channels (ch) & $J_{\text {left }} \times 10^{4}$ & $J_{\text {right }} \times 10^{4}$ & $N /\left(2 J_{\text {right }} \Delta t Q\right)$ & $N /\left[\left(J_{\text {right }}+J_{\text {left }}\right) \Delta t Q\right]$ \\
\hline S2404A & 11.16 & $\begin{array}{c}6026 \\
(30-50 \mathrm{ch})\end{array}$ & $\begin{array}{c}11299 \\
(51-98 \mathrm{ch})\end{array}$ & 1.205 & 1.130 & 0.117 & 0.113 \\
\hline S2404B & 13.93 & $\begin{array}{c}2733 \\
(1-49 \mathrm{ch})\end{array}$ & $\begin{array}{c}11299 \\
(50-110 \mathrm{ch})\end{array}$ & 1.205 & 1.130 & 0.111 & 0.108 \\
\hline S2804A & 8.43 & $\begin{array}{c}5463 \\
(1-26 \mathrm{ch})\end{array}$ & $\begin{array}{c}11511 \\
(27-68 \mathrm{ch})\end{array}$ & 1.091 & 1.155 & 0.110 & 0.113 \\
\hline S2704A & 10.66 & $\begin{array}{c}5755 \\
(1-26 \mathrm{ch})\end{array}$ & $\begin{array}{c}9761 \\
(27-110 \mathrm{ch})\end{array}$ & 1.150 & 0.978 & 0.119 & 0.109 \\
\hline S2804B & 10.78 & $\begin{array}{c}6823 \\
(1-26 \mathrm{ch})\end{array}$ & $\begin{array}{c}13959 \\
(27-110 \mathrm{ch})\end{array}$ & 1.362 & 1.402 & 0.113 & 0.114 \\
\hline S2904A & 12.41 & $\begin{array}{c}5057 \\
(1-26 \mathrm{ch})\end{array}$ & $\begin{array}{c}10626 \\
(27-68 \mathrm{ch})\end{array}$ & 1.009 & 1.067 & 0.111 & 0.114 \\
\hline S2904B & 9.07 & $\begin{array}{c}5926 \\
(1-26 \mathrm{ch})\end{array}$ & $\begin{array}{c}12325 \\
(27-68 \mathrm{ch})\end{array}$ & 1.182 & 1.239 & 0.112 & 0.115 \\
\hline R809A & 10.52 & $\begin{array}{c}20914 \\
(20-79 \mathrm{ch})\end{array}$ & $\begin{array}{c}41141 \\
(80-130 \mathrm{ch})\end{array}$ & 4.154 & 4.170 & 0.0960 & 0.0962 \\
\hline R0909A & 7.12 & $\begin{array}{c}15998 \\
(20-83 \mathrm{ch})\end{array}$ & $\begin{array}{c}31581 \\
(84-130 \mathrm{ch})\end{array}$ & 3.178 & 3.202 & 0.0984 & 0.0987 \\
\hline R1109A & 8.76 & $\begin{array}{c}35021 \\
(20-79 \mathrm{ch})\end{array}$ & $\begin{array}{c}70874 \\
(80-130 \mathrm{ch})\end{array}$ & 6.947 & 7.203 & 0.0907 & 0.0924 \\
\hline R1109B & 8.76 & $\begin{array}{c}14238 \\
(20-84 \mathrm{ch})\end{array}$ & $\begin{array}{c}27711 \\
(85-130 \mathrm{ch})\end{array}$ & 2.821 & 2.822 & 0.103 & 0.103 \\
\hline R1409A & 6.71 & $\begin{array}{c}17119 \\
(54-84 \mathrm{ch})\end{array}$ & $\begin{array}{c}32147 \\
(85-145 \mathrm{ch})\end{array}$ & 3.387 & 3.283 & 0.0982 & 0.0967 \\
\hline R1609A & 9.79 & $\begin{array}{c}9718 \\
(62-82 \mathrm{ch})\end{array}$ & $\begin{array}{c}18913 \\
(83-114 \mathrm{ch})\end{array}$ & 1.943 & 1.892 & 0.102 & 0.101 \\
\hline R1609B & 9.92 & $\begin{array}{c}20714 \\
(20-81 \mathrm{ch})\end{array}$ & $\begin{array}{c}41624 \\
(82-150 \mathrm{ch})\end{array}$ & 4.128 & 4.191 & 0.0831 & 0.0837 \\
\hline $\begin{array}{l}\text { Average, } \\
S \text { files }\end{array}$ & & & & & & $\begin{array}{c}0.113 \\
\pm 0.003\end{array}$ & $\begin{array}{c}0.112 \\
\pm 0.002\end{array}$ \\
\hline Average, & & & & & & 0.0959 & 0.0960 \\
\hline$R$ files & & & & & & \pm 0.0064 & \pm 0.0059 \\
\hline $\begin{array}{l}\text { Average, } \\
\text { without } \mathrm{CH}_{2}\end{array}$ & & & & & & $\begin{array}{c}0.090 \\
\pm 0.009\end{array}$ & $\begin{array}{c}0.090 \\
\pm 0.009\end{array}$ \\
\hline
\end{tabular}

Value error $=0.9068 \pm 0.0005-0.900 \pm 0.010=0.0068$

Table 3 shows the results of using the Rossi- $\alpha$ experiment for the left-hand side of (1) and the result of measurements of the same subcritical assembly by the IKES method (Grabezhnoy et al. 2015).

A similar method was used to determine the subcritical state of a single-core BFS-73 facility, consisting of metallic uranium (18\% enrichment), steel and sodium, in proportions close to the composition of a fast reactor (Dulin VA and Dulin VV 1998).

The multiplication factor, estimated by the standard methods adopted at the BFS facilities (by the IKES method (Kazansky and Matusevich 1994) and by the method of inverse neutron multiplication), was $k=0.90 \pm 0.01$.

The processing of these experiments carried out in (Marchuk and Orlov 1961), taking into account the results of (Mikhailov and Dulin 1995), made it possible to more correctly calculate the space-isotope correlation factor for a medium with a source; however, when calculating $J_{\text {left }}(t)$, the integration of $C_{\text {left }}(t)$ from (2) over $t$ was carried out in the range from 0 to $T$, but not from $-\infty$ to $T$, as follows from (Mikhailov and Dulin 1995). Now this is done by means of integration similar to (2), (3) and (6), (7), analyzing the previously measured files with an accurate knowledge of the delay $T$, but not assuming, as was done in (Grabezhnoy et al. 2015), that it is determined by the position of the peak maximum visible on the screen.

The results of using the Rossi- $\alpha$ experiment and measurements of the subcritical assembly by the IKES method presented in Table 3 are similar to the process described above. Since, in this case, the counters were at the border of the core, and the $\mathrm{Cf}$ sources were 4-10 times smaller than the source of neutrons of spontaneous ${ }^{240} \mathrm{Pu}$ fissions, when files of type $\mathrm{S}$ were measured, covers made of polyethylene $1 \mathrm{~mm}$ thick were put on the counters and, in the case of files of type R, the covers were $4.1 \mathrm{~mm}$ thick.

The data of the IKES method differ insignificantly from the results of the Rossi- $\alpha$ method with an increase in the subcriticality of the assembly (the difference did not exceed the errors). Perhaps, this was due to the simplicity of the geometry of the subcritical single-core BFS-73 facility (Marchuk and Orlov 1961) and, accordingly, the small error in calculating the spatial corrections in both methods. Since in the IKES method, the error in $\beta_{\text {eff }}$ directly enters the error in $k_{\text {eff }}$ (IKES) (as an error in the denominator), and the error in $k_{\text {eff }}($ Rossi- $\alpha)$ - only as an error in the sum $k_{\text {eff }}\left(\varphi_{\sigma}^{+}\right)=k_{\sigma p}+\beta_{\text {eff, }}$ then (if it is true) a small value of the difference $k_{\text {eff }}$ (Rossi- $\left.\alpha\right)-k_{\text {eff }}$ (IKES) indicates a small error in $\beta_{\text {eff }}$ in the IKES method. 


\section{References}

- Dulin VA, Dulin VV (1998) On the measurement of the subcritical states of the breeding media by the Rossi-alpha method. Izvestia Vysshikh Uchebnykh Zavedeniy. Yadernaya Energetika [News of Higher Educational Institutions. Nuclear Power Engineering] 5: 25-28. [in Russian]

- Dulin VA, Matvienko IP (2002) Definition of deep subcriticality of breeding media states by Rossi- $\alpha$ method. Izvestia Vysshikh Uchebnykh Zavedeniy. Yadernaya Energetika [News of Higher Educational Institutions. Nuclear Power Engineering] 1: 9-15. [in Russian]

- Grabezhnoy VA, Dulin VA, Dulin VV (2015) On spatial corrections in Rossi- $\alpha$ experiments. Izvestia Vysshikh Uchebnykh Zavedeniy. Yadernaya Energetika [News of Higher Educational Institutions. Nuclear Power Engineering] 4: 111-123. [in Russian]
Kazansky YuA, Matusevich EC (1994) Experimental Physics of Reactors. Energoatomizdat Publ., Moscow, 116-119. [in Russian]

- Marchuk GI, Orlov VV (1961) On the theory of conjugate functions. In: Neutron Physics. Gosatomizdat Publ., Moscow, 31-34. [in Russian]

- Mikhailov GM, Dulin VA (1995) Measurement of $\beta_{\text {eff }}$ by the Rossi- $\alpha$ Method. Atomnaya Energiya [Nuclear Energy] 78(3): 151-155. [in Russian]

- Takeshi S, Shigeaki O (1970) Experimental core for experiments of effective delayed fraction $\beta_{\text {eff }}$. Progress in Nuclear Energy, Special Issue 35(2): 140-156. https://doi.org/10.1016/S0149-1970(99)00007-4

- Tsutomu I (1968) On the Background Counts in the Rossi-alpha Experiment. Nukleonik, Band 11, Heft 3: 157. 